\title{
Cardiopulmonary Exercise Test in Patients with Hypertension: Focused on Hypertensive Response to Exercise
}

\author{
Jong-Chan Youn Seok-Min Kang \\ Division of Cardiology, Severance Cardiovascular Hospital and Cardiovascular Research \\ Institute, Yonsei University College of Medicine, Seoul, Republic of Korea
}

\section{Key Words}

Cardiopulmonary exercise test $\cdot$ Hypertension

\begin{abstract}
The cardiopulmonary exercise test (CPET) provides integrative exercise responses including the cardiovascular, pulmonary and skeletal muscle systems. It can be used for the identification of myocardial ischemia, evaluation of exercise capacity and tolerance, and the assessment of chronotropic competence or arrhythmias with the addition of ventilatory and gas exchange measurement information. Among them, hypertensive response to exercise (HRE) is known to be related with higher risk of future heart failure and cardiovascular events in patients with hypertension. Proposed underlying mechanisms of HRE can be found in ventricular-vascular uncoupling including decreased aortic distensibility, increased left ventricular mass, endothelial dysfunction, and diastolic dysfunction. The CPET might be useful in the identification of masked hypertension and the assessment of antihypertensive treatment efficacy in patients with hypertension.

(C) 2015 S. Karger AG, Base
\end{abstract}

The cardiopulmonary exercise test (CPET) estimates a broader range of variables related to cardiopulmonary function, including expiratory ventilation and pulmonary gas exchange (oxygen uptake and carbon dioxide output), along with electrocardiography (ECG) and blood pressure (BP), with the goal of quantitatively linking metabolic, cardiovascular, and pulmonary responses to exercise $[1,2]$. The CPET as well as the conventional exercise treadmill test can be used for the provocation and identification of myocardial ischemia, the detection of coronary artery disease in patients with chest pain, the evaluation of physical capacity and exercise tolerance, the evaluation of exercise-related symptoms and the assessment of chro- 
Fig. 1. Proposed mechanisms of HRE in hypertension.

Youn and Kang: Cardiopulmonary Exercise Test in Patients with Hypertension: Focused on Hypertensive Response to Exercise

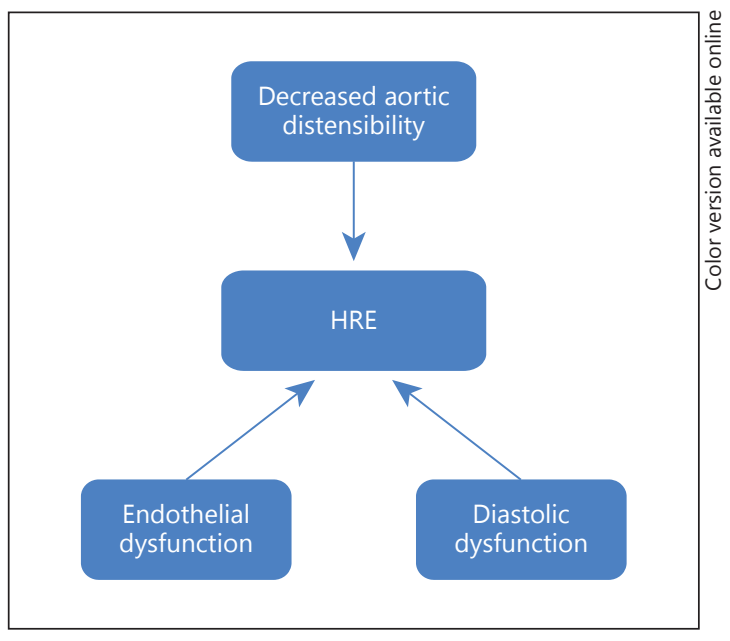

notropic competence and arrhythmias [3]. However, the addition of ventilatory gas exchange measurements to the ECG and BP monitoring used in conventional exercise treadmill tests provides a more accurate determination of the aerobic capacity and a unique insight into the independent and integrated functions of the cardiovascular, pulmonary, and skeletal muscle systems. This mini-review will provide the definition and prognostic value of hypertensive response to exercise (HRE), several proposed mechanisms of HRE, and finally the clinical usefulness of the CPET in patients with hypertension.

Hemodynamic responses to exercise depend on the cardiac output and peripheral resistance. Systolic BP rises with an increasing workload, particularly due to a rise in cardiac output. In contrast, diastolic BP usually remains the same or decreases moderately with exercise. Although there is no widely accepted normal BP response to exercise, an HRE is usually defined as a peak exercise systolic BP of $\geq 210 \mathrm{~mm} \mathrm{Hg}$ in men and $\geq 190 \mathrm{~mm} \mathrm{Hg}$ in women [4]. HRE predicts the future development of essential hypertension [5-8], coronary disease [9], left ventricular (LV) hypertrophy [10], cardiovascular (CV) events [11 12], and mortality [13], independent of a resting BP in apparently healthy, normotensive individuals. Recently, Schultz et al. [14] reported pooled analysis data about the prognostic value of an HRE, based on the data from 46,314 individuals followed up for a mean of 15 years. They demonstrated that an HRE at a moderate exercise workload predicts CV outcomes independently of office $\mathrm{BP}$, age, or multiple CV risk factors. While each $10 \mathrm{~mm} \mathrm{Hg}$ increase in systolic BP during moderate intensity exercise was accompanied by a $4 \%$ increase in CV events and mortality, independent of office BP, age, or CV risk factors (95\% CI, 1.01-1.07, p = 0.02), systolic BP at a maximal workload was not significantly associated with the outcome of an increased rate of $\mathrm{CV}$ [14].

The underlying mechanisms of an exaggerated increase in systolic BP with exercise are not well known. Although the HRE is multifactorial, proposed underlying mechanisms include an excessively high sympathetic tone during exercise, decreased aortic distensibility, increased LV mass as well as endothelial and diastolic dysfunction [15] (fig. 1). Under resting conditions, decreased aortic distensibility, which is closely associated with high BP, is an independent predictor of mortality [14]. A decrease in aortic distensibility during exercise will reduce the buffer capacity of BP and may cause an excessive increase in systolic BP [16, 17]. In addition, structural abnormalities in the peripheral artery or an inability of the peripheral artery to dilate appropriately may result in excessive BP increase during exercise. Indeed, some studies have reported that endothelial dysfunction is related to an HRE [18]. 

Youn and Kang: Cardiopulmonary Exercise Test in Patients with Hypertension: Focused
on Hypertensive Response to Exercise

Moreover, LV diastolic dysfunction might be another underlying contributor of HRE, irrespective of the presence of resting hypertension [19]. Our recent study has shown an independent association between HRE and the left atrial volume index, a marker for chronic diastolic dysfunction in patients with hypertension [20]. HRE was more closely associated with diastolic dysfunction in older ( $>55$ years) hypertensive patients. Aging contributes to diastolic dysfunction accompanied by ventricular and atrial remodeling. An increased left atrial size has been consistently reported in older hypertensive patients, and a left atrial volume index is reported to be a sensitive marker of LV diastolic dysfunction, indicating exercise intolerance. While time intervals and Doppler velocities are mainly influenced by the volume status of the patient at the time of measurements, left atrial volume reflects the cumulative effects of elevated LV filling pressures over a long time. Also, arterial stiffening involving pulse pressure widening, which indirectly represents abnormal vascular function, is suggested as a possible mechanism for HRE.

Taken together, ventricular and vascular stiffening with disordered ventricular-vascular interaction, combined with diastolic dysfunction, might be a potential mechanistic link between an HRE, impaired exercise capacity, and adverse clinical outcomes.

Masked hypertension independently predicts mortality [21] but cannot be easily detected from clinic BP taken under resting conditions. Currently, the methods to identify masked hypertension involve 24-hour ambulatory BP monitoring or home BP monitoring. However, the CPET might be used as a rapid and feasible screening test to identify suspected masked hypertension because masked hypertension could be unmasked via the BP response to light exercise in untreated subjects [22]. During exercise, masked hypertension subjects had a significantly higher systolic BP, with a greater change from baseline. Low-level exercise systolic BP was independently associated with masked hypertension. Another clinical application of the CPET in patients with hypertension can be found in the assessment of antihypertensive treatment efficacy in patients with hypertension. Exercise BP at workloads of 3-5 metabolic equivalents may reflect BP during daily activities. Moreover, various antihypertensive treatments, such as angiotensin receptor antagonist [23] and aldosterone antagonist [24], are known to modulate HRE in patients with hypertension.

In conclusion, the CPET can provide various useful exercise-related parameters in patients with hypertension. Among them, HRE predicts the future development of hypertension, coronary artery disease, LV hypertrophy, heart failure, CV events and mortality. Moreover, the CPET might be useful in the identification of masked hypertension and the assessment of antihypertensive treatment efficacy in patients with hypertension.

\section{Disclosure Statement}

The authors have no conflicts of interest to disclose.

\section{References}

1 Balady GJ, Arena R, Sietsema K, et al: Clinician's Guide to cardiopulmonary exercise testing in adults: a scientific statement from the American Heart Association. Circulation 2010;122:191-225.

$\checkmark 2$ Arena R, Sietsema KE: Cardiopulmonary exercise testing in the clinical evaluation of patients with heart and lung disease. Circulation 2011;123:668-680.

- 3 Fletcher GF, Ades PA, Kligfield P, et al: Exercise standards for testing and training: a scientific statement from the American Heart Association. Circulation 2013;128:873-934.

4 Morrow K, Morris CK, Froelicher VF, et al: Prediction of cardiovascular death in men undergoing noninvasive evaluation for coronary artery disease. Ann Intern Med 1993;118:689-695. 
Youn and Kang: Cardiopulmonary Exercise Test in Patients with Hypertension: Focused on Hypertensive Response to Exercise

5 Manolio TA, Burke GL, Savage PJ, et al: Exercise blood pressure response and 5-year risk of elevated blood pressure in a cohort of young adults: the CARDIA study. Am J Hypertens 1994; 7:234-241.

-6 Singh JP, Larson MG, Manolio TA, et al: Blood pressure response during treadmill testing as a risk factor for new-onset hypertension. The Framingham heart study. Circulation 1999;99:1831-1836.

7 Sharabi Y, Ben-Cnaan R, Hanin A, et al: The significance of hypertensive response to exercise as a predictor of hypertension and cardiovascular disease. J Hum Hypertens 2001;15:353.

-8 Miyai N, Arita M, Miyashita K, et al: Blood pressure response to heart rate during exercise test and risk of future hypertension. Hypertension 2002;39:761-766.

-9 McHam SA, Marwick TH, Pashkow FJ, et al: Delayed systolic blood pressure recovery after graded exercisean independent correlate of angiographic coronary disease. J Am Coll Cardiol 1999;34:754-759.

$\$ 10$ Gottdiener JS, Brown J, Zoltick J, et al: Left ventricular hypertrophy in men with normal blood pressure: relation to exaggerated blood pressure response to exercise. Ann Intern Med 1990;112:161-166.

11 Kurl S, Laukkanen J, Rauramaa R, et al: Systolic blood pressure response to exercise stress test and risk of stroke. Stroke 2001;32:2036-2041.

$\checkmark 12$ Laukkanen JA, Kurl S, Salonen R, et al: Systolic blood pressure during recovery from exercise and the risk of acute myocardial infarction in middle-aged men. Hypertension 2004;44:820-825.

13 Kjeldsen SE, Mundal R, Sandvik L, et al: Supine and exercise systolic blood pressure predict cardiovascular death in middle-aged men. J Hyperten 2001;19:1343-1348.

14 Schultz MG, Otahal P, Cleland VJ, et al: Exercise-induced hypertension, cardiovascular events, and mortality in patients undergoing exercise stress testing: a systematic review and meta-analysis. Am J Hypertens 2013;26: 357-366.

15 Le VV, Mitiku T, Sungar G, et al: The blood pressure response to dynamic exercise testing: a systematic review. Prog Cardiovasc Dis 2008;51:135-160.

-16 O'Rourke MF, Kelly RP: Wave reflection in the systemic circulation and its implications in ventricular function. J Hypertens 1993;11:327-337.

17 Kontsas K, Triantafyllidi H, Trivilou P, et al: Delayed blood pressure recovery ratio might indicate increased arterial stiffness in hypertensive patients with reduced aerobic exercise capacity. Blood Press 2013;22:290296.

18 Stewart KJ, Sung J, Silber HA, et al: Exaggerated exercise blood pressure is related to impaired endothelial vasodilator function. Am J Hypertens 2004;17:314-320.

19 Takamura T, Onishi K, Sugimoto T, et al: Patients with a hypertensive response to exercise have impaired left ventricular diastolic function. Hypertens Res 2008;31:257-263.

20 Lee SE, Youn JC, Lee HS, et al: Left atrial volume index is an independent predictor of hypertensive response to exercise in patients with hypertension. Hypertens Res 2015;38:137-142.

21 Mancia G, Facchetti R, Bombelli M, et al: Long-term risk of mortality associated with selective and combined elevation in office, home, and ambulatory blood pressure. Hypertension 2006;47:846-853.

-22 Schultz MG, Hare JL, Marwick TH, et al: Masked hypertension is 'unmasked' by low-intensity exercise blood pressure. Blood Press 2011;20:284-289.

23 Warner JG Jr, Metzger DC, Kitzman DW, et al: Losartan improves exercise tolerance in patients with diastolic dysfunction and a hypertensive response to exercise. J Am Coll Cardiol 1999;33:1567-1572.

24 Hare JL, Sharman JE, Leano R, et al: Impact of spironolactone on vascular, myocardial, and functional parameters in untreated patients with a hypertensive response to exercise. Am J Hypertens 2013;26:691-699. 